
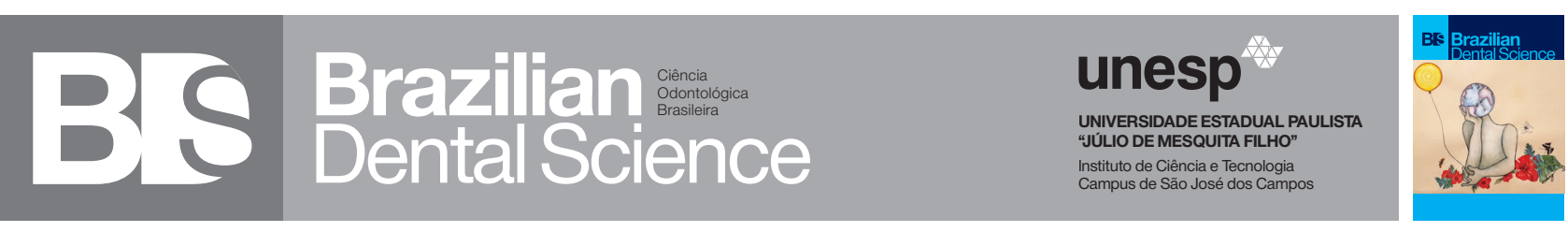

\title{
Comparison of two oral health-related quality-of-life measures in elderly patients: the influence of missing teeth, and socio demographic factors
}

Comparação entre dois instrumentos de avaliação de qualidade de vida em pacientes idosos: a influência da perda de dentes e fatores sociodemográficos

Lígia Moura BURCI ${ }^{1}$, Sandra Maria Warumby ZANIN ${ }^{1}$, Obdúlio Gomes MIGUEL ${ }^{1}$, Astrid Wiens SOUZA ${ }^{1}$, Josiane de Fátima Gaspari DIAS ${ }^{1}$, Marilis Dallarmi MIGUEL ${ }^{1}$

1- Universidade Federal do Paraná (UFPR) - Postgraduate Program in Pharmaceutical Sciences (PPGCF) - Curitiba - PR - Brazil.

\section{ABSTRACT}

Objective: The Oral Health Impact Profile-14 (OHIP14) and the Geriatric/General Oral Health Assessment Index (GOHAI) have never been compared to a group of the same subjects in the Brazilian population. The aim of the study was to compare the OHIP-14 and GOHAI measures. Material and Methods: 129 independently living people over the age of 60 were included in the study. The GOHAI and OHIP-14 measures were used. Other variables were included: age, gender, education, number of missing teeth, annual household income and frequency of dentist visits. Results: The mean age of respondents was 65 years. The internal reliability (Cronbach's alpha) showed a high internal consistency for both measures. Spearman's rank correlation coefficient between the GOHAI and OHIP-14 scores was 0.73 . Using the additive method of creating scores, none of the respondents had the GOHAI score of zero, indicating no impact from oral conditions, while $9.3 \%$ of them had an OHIP-14 score of zero. Dental status, age, gendler and frequency of dental visit were significantly associated with the results ofthe GOHAI and the OHIP-14 (Kruskal-Wallis test, Mann-Whitney U test). Conclusions: There was a strong correlation between the GOHAI and the OHIP14. Both instruments demonstrated good discriminant properties and helped capture the respondents' oral health problems.

\section{KEYWORDS}

Brazil; Older adult; GOHAI; Oral health-related quality of life.

\section{RESUMO}

Objetivo: O Perfil de Impacto na Saúde Oral-14 (OHIP14) e o Índice Geral de Avaliação de Saúde Oral em Geriatria (GOHAI) nunca foram comparados a um grupo dos mesmos sujeitos na população brasileira. O objetivo do estudo foi comparar as medidas OHIP-14 e GOHAI. Material e Métodos: 129 pessoas independentes com idade superior a 60 anos foram incluídas no estudo. Foram utilizadas as medidas GOHAI e OHIP-14. Outras variáveis foram incluídas: idade, sexo, escolaridade, número de dentes ausentes, renda familiar anual e frequência de visitas ao dentista. Resultados: a média de idade dos entrevistados foi de 65 anos. A confiabilidade interna (alfa de Cronbach) mostrou uma alta consistência interna para ambas as medidas. O coeficiente de correlação de Spearman entre os escores GOHAI e OHIP-14 foi de 0,73. Utilizando o método aditivo de criação de escores, nenhum dos entrevistados obteve pontuação zero no GOHAI, indicando nenhum impacto das condições bucais, enquanto 9,3\% deles tiveram pontuação zero no OHIP-14. O estado dentário, a idade, o sexo e a frequência da visita foram significativamente associados aos resultados do GOHAI e do OHIP-14 (teste de Kruskal-Wallis, teste de Mann-Whitney U). Conclusões: Houve uma forte correlação entre o GOHAI e o OHIP-14. Ambos os instrumentos demonstraram boas propriedades discriminantes e ajudaram a capturar os problemas de saúde bucal dos entrevistados.

\section{PALAVRAS-CHAVE}

Brasil; Idosos; GOHAI; Qualidade de vida relacionada à saúde bucal. 


\section{INTRODUCTION}

$\mathrm{T}$ he population of people worldwide is constantly growing older and their health-related quality of life (HRQoL) is an increasing public health concern [1]. The relation between oral health and general health is particularly visible among old people because the large proportion of them does not or even cannot follow the necessary teeth and denture hygiene practices, which has additional negative oral health impacts [2]. Increasing attention is being given to the oral health of older adults because of the age transition of our societies [3]. Poor oral health in older people is particularly reflected in high levels of tooth loss, which in turn may influence general health in terms of weight loss, eating problems, and social disadvantage [4]. Extensive tooth loss reduces chewing performance and limits food choice [5]. The poor appearance of teeth and dentures, and difficulties talking, smiling, and kissing have been associated with social discomfort and embarrassment [6], while tooth loss also has considerable emotional effects [7]. Oral health contributes significantly to the quality of life of adults and affects them physically (e.g. difficulty eating), psychologically, and socially [8].

The outcomes of oral health conditions and therapy for those conditions are described by the term 'oral health-related quality of life' (OHRQoL) [9]. This concept refers to the extent tow hich the oral diseases impact on individuals' normal functioning and is regarded as an integral part of general health and wellbeing. OHRQoL is recognized by the WHO as an important part of the Global Oral Health Program [10]. This is a multidimensional concept that deals with the quality of life (QoL) related to oral health and diseases [10]. The impact of socioeconomic factors on perceptions of health and oral health states have not been frequently investigated [5]. This study applies a Brazilian version of the Geriatric/ General Oral Health Assessment
Index (GOHAI) and Oral Health Impact Profile, short version (OHIP-14) inventory [9] to investigate its relationship with age, other socioeconomic factors and dental status. The present study is based on a conceptual model proposed by Chen \& Hunter [11] suggesting that socioeconomic status, oral health behavior and oral health status each influence OHRQoL.

The Oral Health Impact Profile (OHIP) and the Geriatric/General Oral Health Assessment Index (GOHAI) are regarded as the most comprehensive assessments for measuring the OHRQoL [12]. They have been widely used in research studies on various populations. The measures differ in terms of the item content. Its aim is to assess seven dimensions of impacts of oral conditionson people's OHRQoL including functional limitation, physical pain, psychological discomfort, physical disability,psychological disability, social disability and handicap [13].

The GOHAI measure is a 12-item questionnaire originally developed in 1990 in the USA for use with older adult populations with three months time reference [14]. It was developed to evaluate three dimensionsof oral-health related quality of life, including physical functions like eating, speech, swallowing; psychosocial functions like worry, concern about oral health, dissatisfaction with appearance, self-consciousness about oral health, avoidance of social contacts because of oral problems; pain or discomfort including the use of medication or discomfort from the mouth [14]. According to Locker et al [15], the GOHAI gives a greater weight to functional limitations or pain and discomfort, and the OHIP-14 gives a greater weight to psychological and behavioral outcomes, both describe different aspects of OHRQoL. No comparison study to explore the ability of these two scales has ever been done in Brazil. The aim of the study was to compare the Oral Health ImpactProfile-14 (OHIP-14) and the Geriatric/General Oral Health Assessment 
Index (GOHAI) measures and to assesswhich instrument was more adequate in Brazilian subjects.

\section{METHODS}

\section{Study population}

This study was submitted and approved by the Institutional Review Board regarding ethical aspects (protocol no. \# 954.338). The sample comprised 129 older adult people of the city of Curitiba, aged between 60 and 80 years, attending the health units, at the waiting room for dental or medical care. Participants were recruted by means of the convenience sample in a public dental clinic in Curitiba, the capital of Parana State, in South part of Brazil. The enrollment period was five months (Feb - Jun 2015). The main inclusion criteria was the age of 60 and over. The sample size was based on an a priori assumed correlation between the GOHAI and OHIP scales $[15,16]$.

The participation in the study was both anonymous and voluntary and started after a written consent of the participants. The patients were invited to fill the questionnaire during their regular dental checkup or when they had a requested treatment. The patients who were unable to comprehend the questionnaire were excluded from the study to avoid unreliable answers.

\section{Measures}

The questionnaire used in the survey contained both the GOHAI and the OHIP-14 scales. Three months' reference for the GOHAI and six month's for the OHIP were used. The response format for both on a Likert-type frequency scale was as follows: very often $=3$, occasionally $=2$ and never $=1$ for GOHAI, and: very often $=4$, fairly often $=$ 3 , occasionally $=2$, hardly ever $=1$, never $=0$ [16]. The two measures were compared in terms of their item content. The additive method was used to calculate the GOHAI and the OHIP-14 scores. For the OHIP-14, they were obtained by summing the response codes of the 14 items constituting the measure. Additive scores for the GOHAI were obtained by summing the response codes for the 12 items. Questions were worded positively and negatively to require the participants to consider the answers. The coding of three items like "able to swallow comfortably", "able to eat without discomfort", pleased with the look with teeth" were reversed (high score in the GOHAI indicated a low impairment). Consequently, the GOHAI scale ranged from 10 to 48 and the OHIP-14 scale from 0 to 56 with higher scores indicating a poorer oral health-related quality of life. Other questions referred to age, gender, education and number of missing teeth.

\section{Data analysis}

The Kruskal-Wallis test and the MannWhitney's U test were used to compare the GOHAI and the OHIP-14 scores in relation to gender, education, and number of missing teeth. The GOHAI and the OHIP-14 scores were dichotomized using median splits. Spearman's rank correlation coefficients were used to measure inter-item and itemscore correlations as well as correlations of the GOHAI or OHIP-14 scores with age and number of preserved teeth. The values of Cronbach's alpha were calculated to assess the internal consistency for the whole score and for particular items removed. The statistical analysis was performed using the IBM SPSS Statistics 19.0 software. Statistical hypotheses were verified with a significance level of 0.05 .

\section{RESULTS}

Altogether, the final sample consisted of 129 independently living people, 36 men and 93 women. They ranged in age from 60 to 80, were only $11.6 \%$ were aged 70 and over and the mean age was 65 (SD 7.6) years. As for the educational background, only $39.5 \%$ of subjects declared high school, but the majority had secondary education or less (60.5\%). Furthermore, $60.5 \%$ of the participants have lost more than 5 teeth (Table I). The internal reliability (Cronbach's alpha) was 0.74 for the 
GOHAI and 0.76 for the OHIP-14, showing a high internal consistency. Internal reliability of scales for each single item removed varied between 0.674 (item 10) and 0.768 (item 9) for the GOHAI and 0.708 (item 6) and 0.758 (item 5) for the OHIP-14. The correlation between the GOHAI and OHIP-14 scores using Spearman's rank-correlation coefficient was $0.73(\mathrm{p}<0.001)$.

Table I - Frequency distribution of independent variables and their categories $(n=129)$

\begin{tabular}{|c|c|c|}
\hline Variables & Categories (code) & $N(\%)$ \\
\hline Age (yrs) & $\begin{array}{c}60-70 \\
70-80 \\
80 \text { or more }\end{array}$ & $\begin{array}{c}111(86.0) \\
15(11.6) \\
3(2.3)\end{array}$ \\
\hline Gendler & $\begin{array}{l}\text { Male } \\
\text { Female }\end{array}$ & $\begin{array}{l}36(27.9) \\
93(72.1)\end{array}$ \\
\hline Annual household income & $\begin{array}{l}\text { More than } \$ 7.000 \\
\text { Less than } \$ 7.000\end{array}$ & $\begin{array}{l}48(37.2) \\
81(62.8)\end{array}$ \\
\hline Education level & $\begin{array}{l}\text { More than High school } \\
\text { High school or less }\end{array}$ & $\begin{array}{l}51(39.5) \\
78(60.5)\end{array}$ \\
\hline Dentate status & $\begin{array}{l}<5 \text { missing teeth } \\
5+\text { missing teeth }\end{array}$ & $\begin{array}{l}51(39.5) \\
78(60.5)\end{array}$ \\
\hline Frequency of dental visits & $\begin{array}{l}1+\text { times per year } \\
<1 \text { times per year }\end{array}$ & $\begin{array}{l}78(60.5) \\
51(39.5)\end{array}$ \\
\hline
\end{tabular}

Table II shows the distribution of responses in different dimensions involving the GOHAI instrument. The groups are divided on the loss of teeth, 5 or more missing teeth, or less than 5 missing teeth. In both groups, 5 or more missing teeth and less than 5 missing teeth, the most patients reported difficulty in eating some foods such as meat or apple solid (88.23\% and $61.53 \%$ respectively). As for difficulty swallowing food, patients lost more than 5 teeth reported lower difficulty (58.84\% answered as ever), while those who lost less than 5 teeth reported greater difficulty (42.3\% said always). Most also reported no limit contact with others due to the appearance of your mouth or teeth $(88.23 \%$ and $69.23 \%$ for a loss greater than 5 teeth and a smaller loss of five teeth, respectively), reaching the group of patients who lost more than 5 teeth reporting limit the contact (Table 2).
Table II - Distribution of responses to individual GOHAl items and mean item scores $(n=129)$

\begin{tabular}{|c|c|c|c|c|c|c|}
\hline \multirow{3}{*}{$\begin{array}{l}\text { Dimension and } \\
\text { description of item } \\
\text { The last three } \\
\text { months you: }\end{array}$} & \multicolumn{6}{|c|}{ Distribution of responses \% } \\
\hline & \multicolumn{3}{|c|}{$5+$} & \multicolumn{3}{|c|}{$<5$} \\
\hline & $\begin{array}{l}\text { All the } \\
\text { time } \\
\mathrm{N}(\%)\end{array}$ & $\begin{array}{l}\text { Some- } \\
\text { times } \\
N(\%)\end{array}$ & $\begin{array}{l}\text { Never } \\
\mathrm{N}(\%)\end{array}$ & $\begin{array}{l}\text { All the } \\
\text { time } \\
N(\%)\end{array}$ & $\begin{array}{l}\text { Some- } \\
\text { times } \\
\mathrm{N}(\%)\end{array}$ & $\begin{array}{l}\text { Never } \\
\text { N(\%) }\end{array}$ \\
\hline \multicolumn{7}{|l|}{ Physical functioning } \\
\hline Limit the kins of food & $\begin{array}{c}18 \\
(23.07)\end{array}$ & $\begin{array}{c}12 \\
(15.38)\end{array}$ & $\begin{array}{c}48 \\
(61.53)\end{array}$ & $\begin{array}{c}3 \\
(5.88)\end{array}$ & $\begin{array}{c}3 \\
(5.88)\end{array}$ & $\begin{array}{c}45 \\
(88.24)\end{array}$ \\
\hline $\begin{array}{c}\text { Trouble biting or } \\
\text { hewing }\end{array}$ & $\begin{array}{c}33 \\
(42.30)\end{array}$ & $\begin{array}{c}18 \\
(23.07)\end{array}$ & $\begin{array}{c}27 \\
(34.61)\end{array}$ & $\begin{array}{c}15 \\
(29.41)\end{array}$ & $\begin{array}{c}6 \\
(11.76)\end{array}$ & $\begin{array}{c}30 \\
(58.82)\end{array}$ \\
\hline $\begin{array}{l}\text { Unable to speak } \\
\text { clearly }\end{array}$ & $\begin{array}{c}54 \\
(69.23)\end{array}$ & $\begin{array}{c}6 \\
(7.69)\end{array}$ & $\begin{array}{c}18 \\
(23.08)\end{array}$ & $\begin{array}{c}36 \\
(70.58)\end{array}$ & $\begin{array}{c}6 \\
(11.76)\end{array}$ & $\begin{array}{c}9 \\
(17.65)\end{array}$ \\
\hline \multicolumn{7}{|l|}{ Pain and discomfort } \\
\hline $\begin{array}{l}\text { Able to swallow } \\
\text { comfortably }\end{array}$ & $\begin{array}{c}12 \\
(15.38)\end{array}$ & $\begin{array}{c}27 \\
(34.61)\end{array}$ & $\begin{array}{c}39 \\
(50.0)\end{array}$ & $\begin{array}{c}9 \\
(17.65)\end{array}$ & $\begin{array}{c}15 \\
(29.41)\end{array}$ & $\begin{array}{c}27 \\
(52.94)\end{array}$ \\
\hline $\begin{array}{l}\text { Able to eat without } \\
\text { discomfort }\end{array}$ & $\begin{array}{c}45 \\
(57.69)\end{array}$ & $\begin{array}{c}18 \\
(23.07)\end{array}$ & $\begin{array}{c}15 \\
(19.23)\end{array}$ & $\begin{array}{c}33 \\
(64.71)\end{array}$ & $\begin{array}{c}12 \\
(23.53)\end{array}$ & $\begin{array}{c}6 \\
(11.76)\end{array}$ \\
\hline $\begin{array}{l}\text { Use medication to } \\
\text { relieve pain }\end{array}$ & $\begin{array}{c}3 \\
(3.85)\end{array}$ & $\begin{array}{c}21 \\
(26.92)\end{array}$ & $\begin{array}{c}54 \\
(69.23)\end{array}$ & $\begin{array}{c}0 \\
(0)\end{array}$ & $\begin{array}{c}0 \\
(0)\end{array}$ & $\begin{array}{c}45 \\
(88.23)\end{array}$ \\
\hline $\begin{array}{l}\text { Sensitive to hot, cold } \\
\text { or sweet foods }\end{array}$ & $\begin{array}{c}24 \\
(30.77)\end{array}$ & $\begin{array}{c}12 \\
(15.38)\end{array}$ & $\begin{array}{c}42 \\
(53.85)\end{array}$ & $\begin{array}{c}12 \\
(23.53)\end{array}$ & $\begin{array}{c}0 \\
(0)\end{array}$ & $\begin{array}{c}39 \\
(76.47)\end{array}$ \\
\hline \multicolumn{7}{|c|}{ Psychosocial functioning } \\
\hline $\begin{array}{l}\text { Limit contact with } \\
\text { people }\end{array}$ & $\begin{array}{c}3 \\
(3.85)\end{array}$ & $\begin{array}{c}9 \\
(11.53)\end{array}$ & $\begin{array}{c}66 \\
(84.62)\end{array}$ & $\begin{array}{c}9 \\
(17.65)\end{array}$ & $\begin{array}{c}9 \\
(17.65)\end{array}$ & $\begin{array}{c}33 \\
(64.71)\end{array}$ \\
\hline $\begin{array}{l}\text { Pleased with look } \\
\text { of teeth }\end{array}$ & $\begin{array}{c}27 \\
(34.62)\end{array}$ & $\begin{array}{c}27 \\
(34.61)\end{array}$ & $\begin{array}{c}24 \\
(30.77)\end{array}$ & $\begin{array}{c}27 \\
(52.94)\end{array}$ & $\begin{array}{c}24 \\
(47.05)\end{array}$ & $\begin{array}{c}0 \\
(0)\end{array}$ \\
\hline $\begin{array}{l}\text { Worried about teeth, } \\
\text { gums or dentures }\end{array}$ & $\begin{array}{c}36 \\
(46.15)\end{array}$ & $\begin{array}{c}15 \\
(19.23)\end{array}$ & $\begin{array}{c}33 \\
(42.30)\end{array}$ & $\begin{array}{c}30 \\
(58.82)\end{array}$ & $\begin{array}{c}3 \\
(5.88)\end{array}$ & $\begin{array}{c}18 \\
(35.29)\end{array}$ \\
\hline $\begin{array}{l}\text { Self-conscious } \\
\text { of teeth, gums or } \\
\text { dentures }\end{array}$ & $\begin{array}{c}21 \\
(26.92)\end{array}$ & $\begin{array}{c}21 \\
(26.92)\end{array}$ & $\begin{array}{c}42 \\
(53.85)\end{array}$ & $\begin{array}{c}12 \\
(23.53)\end{array}$ & $\begin{array}{c}3 \\
(5.88)\end{array}$ & $\begin{array}{c}36 \\
(70.58)\end{array}$ \\
\hline $\begin{array}{l}\text { Uncomfortable } \\
\text { eating in front of } \\
\text { others }\end{array}$ & $\begin{array}{c}27 \\
(34.61)\end{array}$ & $\begin{array}{c}6 \\
(18.18)\end{array}$ & $\begin{array}{c}30 \\
(38.46)\end{array}$ & $\begin{array}{c}18 \\
(35.29)\end{array}$ & $\begin{array}{c}9 \\
(17.65)\end{array}$ & $\begin{array}{c}24 \\
(30.77)\end{array}$ \\
\hline
\end{tabular}

The most commonly reported oral health quality of life impacts in OHIP-14 was within the dimension 'psychological discomfort' (Table III). It was found that $19.23 \%$ of subjects who lost 5 or more teeth $(5+)$ found it uncomfortable to eat any foods 'fairly often' or 'very often' compared to $5.88 \%$ who has lost teeth or lost less than $5(<5)$. Nearly $46.15 \%$ of $5+$ reported being self-conscious 'fairly/very often' compared to $47.06 \%$ in the $<5$, while $23.08 \%$ of $5+$ reported that her diet has been unsatisfactory 'fairly/ very often' compared to $5.88 \%$ in the $<5$. In addition, $30.77 \%$ of $5+$ compared to $23.53 \%$ of $<5$ reported impacts 'fairly/very often' with regards to difficulty pronouncing words. Interestingly, when asked 
about the difficulty in relaxing the subject $5+$ showed $23.8 \%$ 'fairly/ very often' compared to $41.18 \%$ in the $<5$.

Table III - Distribution of responses to individual OHIP-14 items and mean item scores $(n=129)$

\begin{tabular}{|c|c|c|c|c|c|c|}
\hline \multirow{3}{*}{$\begin{array}{l}\text { Dimension and } \\
\text { description of item } \\
\text { "Because of } \\
\text { trouble with your } \\
\text { teeth, mouth or } \\
\text { dentures during } \\
\text { the last year,..." }\end{array}$} & \multicolumn{6}{|c|}{ Distribution of responses $\mathrm{N}(\%)$} \\
\hline & \multicolumn{2}{|c|}{$\begin{array}{c}\text { Never (0)/Hardly } \\
\text { Ever (1) }\end{array}$} & \multicolumn{2}{|c|}{ Occasionally (2) } & \multicolumn{2}{|c|}{$\begin{array}{c}\text { Fairly Often (3)/ } \\
\text { Very Often (4) }\end{array}$} \\
\hline & $5+$ & $<5$ & $5+$ & $<5$ & $5+$ & $<5$ \\
\hline \multicolumn{7}{|l|}{ Functional limitation } \\
\hline $\begin{array}{l}\text { have you had trouble } \\
\text { pronouncing any } \\
\text { words? }\end{array}$ & $\begin{array}{c}48 \\
(61.53)\end{array}$ & $\begin{array}{c}33 \\
(64.70)\end{array}$ & $\begin{array}{c}6 \\
(7.69)\end{array}$ & $\begin{array}{c}6 \\
(11.76)\end{array}$ & $\begin{array}{c}24 \\
(30.77)\end{array}$ & $\begin{array}{c}12 \\
(23.53)\end{array}$ \\
\hline $\begin{array}{l}\text { have you felt that } \\
\text { your sense of taste } \\
\text { has worsened? }\end{array}$ & $\begin{array}{c}50 \\
(64.10)\end{array}$ & $\begin{array}{c}45 \\
(88.23)\end{array}$ & $\begin{array}{c}6 \\
(7.69)\end{array}$ & $\begin{array}{c}3 \\
(5.88)\end{array}$ & $\begin{array}{c}21 \\
(26.92)\end{array}$ & $\begin{array}{c}3 \\
(5.88)\end{array}$ \\
\hline \multicolumn{7}{|l|}{ Physicalpain } \\
\hline $\begin{array}{l}\text { have you had painful } \\
\text { aching in your } \\
\text { mouth? }\end{array}$ & $\begin{array}{c}72 \\
(92.31)\end{array}$ & $\begin{array}{c}45 \\
(88.23)\end{array}$ & $\begin{array}{l}0 \\
(0)\end{array}$ & $\begin{array}{c}3 \\
(5.88)\end{array}$ & $\begin{array}{c}6 \\
(7.69)\end{array}$ & $\begin{array}{c}3 \\
(5.88)\end{array}$ \\
\hline $\begin{array}{l}\text { have you found it } \\
\text { uncomfortable to eat } \\
\text { any foods? }\end{array}$ & $\begin{array}{c}51 \\
(65.38)\end{array}$ & $\begin{array}{c}42 \\
(82.35)\end{array}$ & $\begin{array}{c}12 \\
(15.38)\end{array}$ & $\begin{array}{c}6 \\
(11.76)\end{array}$ & $\begin{array}{c}15 \\
(19.23)\end{array}$ & $\begin{array}{c}3 \\
(5.88)\end{array}$ \\
\hline \multicolumn{7}{|c|}{ Psychological discomfort } \\
\hline $\begin{array}{l}\text { have you been } \\
\text { self-conscious? }\end{array}$ & $\begin{array}{c}36 \\
(46.15)\end{array}$ & $\begin{array}{c}21 \\
(41.17)\end{array}$ & $\begin{array}{c}6 \\
(7.69)\end{array}$ & $\begin{array}{c}6 \\
(11.76)\end{array}$ & $\begin{array}{c}36 \\
(46.15)\end{array}$ & $\begin{array}{c}24 \\
(47.06)\end{array}$ \\
\hline have you felt tense? & $\begin{array}{c}26 \\
(33.3)\end{array}$ & $\begin{array}{c}18 \\
(35.29)\end{array}$ & $\begin{array}{c}15 \\
(19.23)\end{array}$ & $\begin{array}{c}15 \\
(29.41)\end{array}$ & $\begin{array}{c}27 \\
(34.62)\end{array}$ & $\begin{array}{c}18 \\
(35.29)\end{array}$ \\
\hline \multicolumn{7}{|l|}{ Physical disability } \\
\hline $\begin{array}{l}\text { has your diet been } \\
\text { unsatisfactory? }\end{array}$ & $\begin{array}{c}54 \\
(69.23)\end{array}$ & $\begin{array}{c}45 \\
(88.23)\end{array}$ & $\begin{array}{c}6 \\
(7.69)\end{array}$ & $\begin{array}{c}3 \\
(5.88)\end{array}$ & $\begin{array}{c}18 \\
(23.08)\end{array}$ & $\begin{array}{c}3 \\
(5.88)\end{array}$ \\
\hline $\begin{array}{l}\text { have you had to } \\
\text { interrupt meals? }\end{array}$ & $\begin{array}{c}69 \\
(88.46)\end{array}$ & $\begin{array}{c}4 \\
(7.84)\end{array}$ & $\begin{array}{l}0 \\
(0)\end{array}$ & $\begin{array}{c}9 \\
(17.65)\end{array}$ & $\begin{array}{c}9 \\
(11.54)\end{array}$ & $\begin{array}{l}0 \\
(0)\end{array}$ \\
\hline \multicolumn{7}{|c|}{ Psychological disability } \\
\hline $\begin{array}{l}\text { have you found it } \\
\text { difficult to relax? }\end{array}$ & $\begin{array}{c}48 \\
(61.53)\end{array}$ & $\begin{array}{c}30 \\
(58.82)\end{array}$ & $\begin{array}{c}12 \\
(15.38)\end{array}$ & $\begin{array}{c}0 \\
(0)\end{array}$ & $\begin{array}{c}18 \\
(23.08)\end{array}$ & $\begin{array}{c}21 \\
(41.18)\end{array}$ \\
\hline $\begin{array}{l}\text { have you been a bit } \\
\text { embarrassed? }\end{array}$ & $\begin{array}{c}36 \\
(46.15)\end{array}$ & $\begin{array}{c}24 \\
(47.05)\end{array}$ & $\begin{array}{c}6 \\
(7.69)\end{array}$ & $\begin{array}{c}3 \\
(5.88)\end{array}$ & $\begin{array}{c}36 \\
(46.15)\end{array}$ & $\begin{array}{c}24 \\
(47.06)\end{array}$ \\
\hline \multicolumn{7}{|l|}{ Social disability } \\
\hline $\begin{array}{c}\text { have you been a bit } \\
\text { irritable with other } \\
\text { people? }\end{array}$ & $\begin{array}{c}42 \\
(53.85)\end{array}$ & $\begin{array}{c}27 \\
(52.94)\end{array}$ & $\begin{array}{c}6 \\
(7.69)\end{array}$ & $\begin{array}{c}3 \\
(5.88)\end{array}$ & $\begin{array}{c}30 \\
(38.46)\end{array}$ & $\begin{array}{c}21 \\
(41.18)\end{array}$ \\
\hline $\begin{array}{l}\text { have you had difficul- } \\
\text { ty doing your usual } \\
\text { jobs? }\end{array}$ & $\begin{array}{c}72 \\
(92.31)\end{array}$ & $\begin{array}{c}36 \\
(70.59)\end{array}$ & $\begin{array}{c}3 \\
(3.85)\end{array}$ & $\begin{array}{c}6 \\
(11.76)\end{array}$ & $\begin{array}{c}3 \\
(3.85)\end{array}$ & $\begin{array}{c}9 \\
(17.65)\end{array}$ \\
\hline \multicolumn{7}{|l|}{ Handicap } \\
\hline $\begin{array}{l}\text { have you felt that life } \\
\text { in general was less } \\
\text { satisfying? }\end{array}$ & $\begin{array}{c}45 \\
(57.69)\end{array}$ & $\begin{array}{c}39 \\
(76.47)\end{array}$ & $\begin{array}{c}9 \\
(11.54)\end{array}$ & $\begin{array}{c}6 \\
(11.76)\end{array}$ & $\begin{array}{c}24 \\
(30.74)\end{array}$ & $\begin{array}{c}6 \\
(11.76)\end{array}$ \\
\hline $\begin{array}{l}\text { have you been totally } \\
\text { unable to function? }\end{array}$ & $\begin{array}{c}72 \\
(92.31)\end{array}$ & $\begin{array}{c}42 \\
(82.35)\end{array}$ & $\begin{array}{l}0 \\
(0)\end{array}$ & $\begin{array}{l}0 \\
(0)\end{array}$ & $\begin{array}{c}6 \\
(7.69)\end{array}$ & $\begin{array}{c}9 \\
(17.65)\end{array}$ \\
\hline
\end{tabular}

Table IV shows the percentage of participants who responded very often, fairly often, occasionally or hardly ever to each GOHAI and OHIP-14 item. Four sub-scales were created for each measure using the domains and items. Using GOHAI ADD scores, 3.85\% reported no functional limitations, $3.85 \%$ no pain or discomfort, $1.93 \%$ no psychological impacts and $63.47 \%$ no behavioral impacts. The corresponding statistics when OHIP-14 ADD scores were used were $44.19 \%, 58.34 \%$, $13.96 \%$ and $27.91 \%$, for the same outcomes respectively.

None of the subjects scored the maximum in either measure. The GOHAI score ranged from 10 to 45 and the OHIP-14 score ranged from 0 to 41 . We found that none participant did report any issues on the GOHAI scale, and 12 on the OHIP-14 scale. Using the additive method of creating scores, none had the GOHAI score of 0 , indicating no impact from oral conditions, while 9.3\% had an OHIP-14 score of 0 . Differences in the distributions of the GOHAI and the OHIP-14 scores were also reflected in their median values of 32 and 16, respectively.

Table IV - Percentage of subjects responding sometimes, fairly often, very often or all the time to each GOHAl and OHIP-14 item

\begin{tabular}{|cccc|}
\hline GOHAl & $\%$ & OHIP-14 & $\%$ \\
\hline Functionallimitation & 96.15 & & 55.81 \\
\hline Trouble biting/chewing food & 55.8 & Trouble pronouncing words & 41.9 \\
\hline Uncomfortable to swallow & 79.1 & Sense of taste worse & 27.9 \\
\hline Prevented from speaking & 48.8 & & \\
\hline Pain and discomfort & 96.15 & & 41.66 \\
\hline Discomfort when eating & 83.8 & Painful aching in mouth & 16.3 \\
\hline Use medication to relieve pain & 23.3 & Uncomfortable to eat foods & 30.2 \\
\hline Teeth, gums, sensitive to hot/cold & 58.1 & & \\
\hline Psychological impacts & 98.07 & & 86.04 \\
\hline Unhappy with appearance & 37.2 & Been self-conscious & 58.1 \\
\hline Worried or concerned & 81.4 & Felt tense & 67.4 \\
\hline Nervous or self-conscious & 60.5 & Difficult to relax & 32.6 \\
\hline Uncomfortable eating in front of people & 39.5 & Been embarrassed & 23.3 \\
\hline Behavioral impacts & & Felt life is less satisfying & 51.2 \\
\hline Limit kinds or amounts of food & 36.53 & & 72.09 \\
\hline Limit contact with others & 27.9 & Diethas been unsatisfactory & 60.5 \\
\hline & & Had to interrupt meals & 55.8 \\
\hline & & Been irritable with others & 30.2 \\
\hline & & Difficulty doing usual jobs & 37.2 \\
\hline & & Totally unable to function & 18.6 \\
\hline
\end{tabular}


Table $\mathrm{V}$ shows the mean values of the GOHAI and OHIP-14 and particular grouping variables. Both measures showed significant associations with being dentate and edentulous, gender, age and frequency of dental visits. Annual household income and schoolarity, did not show any significant relation to the GOHAI and OHIP- 14 scores.

Table V - Mean values of the GOHAl and the OHIP-14 scores of grouping variables

\begin{tabular}{|c|c|c|}
\hline & GOHAIMean (SD) & OHIP-14 Mean(SD) \\
\hline \multicolumn{3}{|l|}{ Age } \\
\hline $60-70$ & $30.16(0.89)$ & $16.03(0.94)$ \\
\hline $70-80$ & $35.6(1.70)$ & $9.00(2.16)$ \\
\hline 80 or more & $30.00(0.0)$ & $24(0.0)$ \\
\hline $\mathrm{p}$ & 0.033 & 0.019 \\
\hline \multicolumn{3}{|l|}{ Gender } \\
\hline Male & $34.00(1.12)$ & $13.60(1.28)$ \\
\hline Female & $30.00(0.96)$ & $17.39(0.98)$ \\
\hline$p$ & 0.027 & 0.036 \\
\hline \multicolumn{3}{|l|}{ Annual household income } \\
\hline More than $\$ 7.000$ & $30.38(1.25)$ & $13.00(1.05)$ \\
\hline Less than $\$ 7.000$ & $31.04(1.06)$ & $17.44(1.21)$ \\
\hline$p$ & 0.690 & 0.013 \\
\hline \multicolumn{3}{|l|}{ Education level } \\
\hline More than High school & $29.13(1.13)$ & $15.60(1.47)$ \\
\hline High school or less & $32.75(0.93)$ & $16.35(1.16)$ \\
\hline$p$ & 0.015 & 0.694 \\
\hline \multicolumn{3}{|l|}{ Dentate status } \\
\hline$<5$ missing teeth & $33.38(1.10)$ & $13.44(1.17)$ \\
\hline $5+$ missing teeth & $29.69(1.09)$ & $16.90(1.18)$ \\
\hline$p$ & 0.026 & 0.047 \\
\hline \multicolumn{3}{|l|}{ Frequency of dental visits } \\
\hline$<1$ times per year & $25.40(1.10)$ & $17.53(1.48)$ \\
\hline $1+$ times per year & $30.15(1.05)$ & $13.76(0.97)$ \\
\hline$p$ & 0.011 & 0.028 \\
\hline
\end{tabular}

\section{DISCUSSION}

The GOHAI and OHIP-14 were selected for this study because they are short and unlikely to entail significant respondent burden and have been widely used worldwide in studies of older adult populations.

Our study is the first conducted in Brazil comparing the GOHAI and the OHIP-14 among the same older adult participants. At least 80 articles have been published in Brazil using the OHIP-14 questionnaire, among them we can mention a study of chronic periodontal disease and its impact on quality of life [17], social relations, economic and behavioral associated with the quality of life and oral health [18] and loss of teeth impact on quality of life, assessed in adult [19].

The GOHAI tool was applied to research in Brazil in at least 18 studies, highlighting the comparison between older adult patients with Alzheimer's [20], in two different regions in Brazil [21] and another dealing with institutionalized older adult [19].

Both tools are recognized instruments for the evaluation of the oral health-related quality of life in the adults and older adult population in relation to objectively measured oral functions. The GOHAI and OHIP-14 differ in the items content and time references. However, the purpose of this study was to compare the GOHAI and OHIP-14 in terms of oral health. Therefore, it is beneficial that there were no other burdens and/or significant differences among participants, with the exception of oral health. Persons who exhibited compromised cognitive function were not included in this group because their answers may have been less reliable. Therefore, the selected study population was thought to be appropriate for the objectives of this study, this being evaluated by the responsible for the interviews if the patient had trouble remembering his name and address.

The participants in our study were clinical seniors who participated in the public dental sector (SUS). Studies show that in the state of Parana, about $10.1 \%$ of seniors reported never having attended by a dentist, and also according to Martins et al [22], the South region of Brazil is the one with the highest percentage in dentate older adults when compared to other regions of the country.

Several studies have shown that the use of dental services decreases with age, reaching the lowest rates to $60 / 65$ years [22]. 
These results were confirmed in a previous study developed in Brazil, using the National Household Survey database 1998 [23]. Results of Martins et al [22], show that more than a half of Brazilians older adults need dental work, which may reflect the actual need for treatment among older Brazilians adults, since the country has the highest prevalence of edentulism and a portion of edentulous without denture.

The level of the OHRQoL in the population is expressed by the number of subjects with a score of 0 in the particular measure. A substantial proportion of older adults respondents showed many problems related to oral health. We found only some individuals with a high OHRQoL, a score of 0 was only found in $9.3 \%$ for the OHIP- 14 . Our findings that the number of respondents who scored 0 in GOHAI was lower than OHIP14 are in accordance with other reports. In Rodakowska et al [24], the score of 0 was $1.1 \%$ for the GOHAI and $13.5 \%$ for the OHIP14.

It has to be emphasized that, although the mean age of our participants was at least about 70, mostly they lost more than 5 teeth. They definitely needed full or partial dentures to sustain the basic oral functions. A threshold of 20 teeth is regarded as a functional and nutritional adequacy of dentition [25]. It should be noted that, at present, for much of the older adult population, the solution to oral health problems should be inserting dentures [26]. According to Gerristen et al [27], tooth loss is strongly associated with the OHRQoL as a more negative impact, and complete or almost complete dentition is associated with the best oral health related quality of life.

In Brazil, the GOHAI score over 30 was also associated with lost teeth [28], which is according to our study, showing a significant difference between the mean values of GOHAI between groups lost more or less than five teeth. It is not surprising that a number of teeth had the strongest association with OHRQoL.
In addition, older subjects had better OHRQoL by GOHAI after controlling for the number of teeth, which is similar to the findings reported by Steele et al [26] that adults aged 70 years and older showed much better OHRQoL when compared with adults with less than 70 years.

These results show that the Brazilian older adults have a low rate use of dental services. Taken together, the media of visit rate to the dentist less than one year $(13.2 \%)$ is about three times smaller that observed for American older adult population [22]. Studies conducted in developed countries have shown that differences in the use of health services for men and women decreases with age, which is not a factor determining this use in older ages. In the present work, there was no association between visit the dentist and sex.

The baseline of the problems reported in the surveys on OHRQoL could differ in particular countries due to such factors as the affluence of the society or the educational level. In the general expectations and life experience of people living in developed countries indicate that it is possible to have full dentition in older adult age and avoid dentures [28].

The GOHAI and the OHIP-14 emphasize items that assess functional limitations and pain, and those showing psychological and behavioral impacts. "Behavioral impacts" health domain being the least frequently reported in our study in the GOHAI and the OHIP-14 measure is consistent with other studies conducted in Canada, Japan and Polish $[15,16]$.

In Brazilian respondents the most frequently reported item was "psychological impacts" in both GOHAI and OHIP, but in Polish and Japan psychological impacts is the most reported only in OHIP, whereas pain and discomfort in GOHAI [16], and in the study of Locker et al [15] "functional limitations" were the most frequently reported items.

Although the oral health status impact on various aspects of quality of life for 
this group, social disability dimension, which refers to social interaction and the development of routine activities, the damage observed, represented by the lowest score achieved. It was much lower than for the other dimensions. In this study, it was found that the aspects that bother them edentulous patients are the shame of not having teeth - dimension of psychological disability - the hassle to eat - dimension pain - and the concern with the mouth dimension psychological discomfort - and impaired power - dimension physical disability.

Our results showed that both, the GOHAI and the OHIP-14 detected the impacts of oral disorders in the evaluated Brazilian population. However, differences could be observed between the GOHAI and the OHIP14 in terms of discriminating the oral healthrelated quality of life outcomes. The value of 0.73 using Spearman's rank-correlation coefficient indicates a strong correlation between the GOHAI and OHIP-14 scores, suggesting that both the OHIP-14 and GOHAI reflect the OHRQoL in a similar manner, and that result as similar when compared with all mentioned Canadian (0.73), Japanese (0.728), German ( $>0.8$ ), Polish (0.81) and in Lebanes studies $[15,16]$. According to Hassel et al [29], in case of assessing a broader concept of the OHRQoL, the OHIP-14 should be chosen. We also proved a higher internal reliability (Cronbach's alpha) for the OHIP-14 compared to the GOHAI, due to its better internal consistency. This can be partly explained by the fact that the OHIP-14 has more items than the GOHAI and according to Locker et al [15] it is also a more homogenous measure with the majority of psychosocial outcomes. Studies conducted by Locker et al [15], Hassel et al [29], Ikebe et al [16], Rodakowska et al [24] and Osta et al [30] also showed that the GOHAI was more successful in detecting older adult people's oral health problems. According to Locker et al [15], the GOHAI gives a greater weight to the more immediate outcomes like functional limitations and pain and discomfort, and therefore more common outcomes of oral disorders compared to the OHIP-14 which focuses on more severe and less common, like psychological and behavioral outcomes. In our study the GOHAI showed an impairment that was not reflected by the OHIP-14. Consequently, the studies that assessed only one measure, either the OHIP-14 or the GOHAI, did not show the full spectrum of the problem.

Our study obviously has some limitations. First, the wide range of participants age being 60 and over could be considered as a limitation. We decided to set, the inclusion criterion at the age of 60 because both measure were recently used in various age group [28]. Another limitation is that the data were gathered by means of a convenience sample from individuals who attended a public dental clinic.

There was a strong correlation between the GOHAI and the OHIP-14. Dental status, chewing ability and self-related oral health in the evaluated Brazilian older adult group was strongly associated with problems identified using the GOHAI and the OHIP-14. In our study both instruments demonstrated good discriminant properties and helped capture the respondents oral health problems.

\section{CONFLICT OF INTERESTS}

The authors have not declared any conflict of interest.

\section{REFERENCES}

1. Liu N, Zeng L, LiZ, Wang J. Health-related quality of life and long-term care needs among elderly individuals living alone: a cross-sectional study in rural areas of Shaanxi Province, China. BMC Public Health. 2013 Apr 8;13:313. doi: 10.1186/1471-2458-13-313

2. Divaris K, Ntounis A, Marinis A, Polyzois G, Polychronopoulou A. Loss of natural dentition: multi-level effects among a geriatric population. Gerodontology. 2012 Jun;29(2):e192-9. doi: 10.1111/j.1741-2358.2010.00440.x.

3. Locker D. Applications of self-reported assessments of oral health outcomes. JDentEduc. 1996 Jun;60(6):494-500.

4. Sheiham A, Maizels JE, Cushing AM. The concept of need in dental care. Int Dent J. 1982 Sep;32(3):265-70. 
5. Allen PF.Assessment of oral health related quality of life. Health Qual Life Outcomes.2003 Sep 8;:1:40.

6. Locker D. Measuring oral health: a conceptual framework. Community Dent Health. 1988 Mar;5(1):3-18.

7. Montero J,Bravo M, Albaladejo A. Validation of two complementary oral-health related quality of life indicators (OIDP and OSS 0-10) in two qualitatively distinct samples of the Spanish population. Health Qual Life Outcomes. 2008 Nov 18;6:101. doi: 10.1186/1477-7525-6-101

8. Montero-Martín J,Bravo-Pérez M, Albaladejo-Martínez A, Hernández-Martín LA, Rosel-Gallardo EM. Validation the Oral Health ImpactProfile (OHIP-14sp) for adults in Spain. Med Oral Patol Oral Cir Bucal. 2009 Jan 1;14(1):E44-50.

9. Locker D, Allen F. What do measures of 'oral health-related quality of life' measure? Community Dent Oral Epidemiol. 2007 Dec;35(6):401-11.

10. Sischo L, Broder HL. Oral health-related quality of life: what, why, how, and future implications. JDent Res. 2011Nov;90(11):1264-70. doi: $10.1177 / 0022034511399918$

11. Chen MS, Hunter P.Oral health and quality of life in New Zealand: a social perspective. Soc Sci Med. 1996 0ct;43(8):1213-22.

12. Locker D, Gibson B. Discrepancies between self-ratings of and satisfaction with oral health in two older adult populations. Community Dent Oral Epidemiol. 2005 Aug;33(4):280-8.

13. Slade GD, Spencer AJ. Development and evaluation of the Oral Health Impact Profile.Community Dent Health. 1994 Mar;11(1):3-11.

14. Atchison KA, Dolan TA. Development of the Geriatric Oral Health Assessment Index.J JentEduc. 1990 Nov;54(11):680-7.

15. Locker D, Matear D, Stephens M, Lawrence H, Payne B. Comparison of the GOHAl and OHIP-14 as measures of the oral health-related quality of life of the elderly. Community Dent Oral Epidemiol.20010ct;29(5):373-81.

16. Ikebe K, Hazeyama T,Enoki K, Murai S, Okada T,Kagawa R, etal. Comparison of GOHAl and OHIP-14 measures in relation to objective values of oral function in elderly Japanese. Community Dent Oral Epidemiol. 2012 Oct:40(5):406-14. doi: 10.1111/j.1600-0528.2012.00683.x

17. Slade GD. Derivation and validation of a short-form oral health impact profile. Community Dent Oral Epidemiol. 1997 Aug;25(4):284-90

18. Gabardo MCL, Moysés SJ, Moysés ST, Olandoski M, Olinto MTA, Pattussi MP.Social, economic, and behavioral variables associated with oral healthrelated quality of life among Brazilian adults. Ciência \& Saúde Coletiva. 2015

\section{0(5):1531-40. doi:https://doi.org/10.1590/1413-81232015205.13562014}

19. Vasconcelos LCA, Prado Júnior RR, Teles JBM, Mendes RF. Autopercepção da saúde bucal de idosos de um munícipio de médio porte do Nordeste brasileiro. Cad Saúde Pública 2012:28(6):1101-1110. doi:https://doi.org/101590/S0102311X2012000600009

20. Batista MJ, Lawrence HP, de Sousa Mda L Impact of tooth loss related to number and position on oral health quality of life among adults. Health Qual Life Outcomes. 2014 Nov 30;12:165. doi: 10.1186/s12955-014-0165-5.

21. Ribeiro GR, Costa JL, Ambrosano GM, Garcia RC. Oral health of the elderly with Alzheimer's disease. Oral Surg Oral Med Oral Pathol Oral Radiol. 2012 Sep;114(3):338-43. doi: 10.1016/j.0000.2012.03.028.

22. Martins AMEBL, Barreto SM, Pordeus IA. Uso de serviços odontológicos entre idosos brasileiros. Rev Panam Salud Publica. 2007.22: 308-16.

23. Strayer MS, Kuthy RA, Caswell RJ, Moeschberger ML. Predictors of dental use for low-income, urban elderly persons upon removal of financial barriers. Gerontologist. 1997 Feb;37(1):110-6.

24. Rodakowska E, MierzyńskaK, Bagińska J, Jamiołkowski J. Quality of life measured by OHIP-14 and GOHAl in elderly people from Bialystok, north-east Poland. BMC Oral Health. 2014 Aug 20;14:106. doi: 10.1186/1472-6831-14-106.

25. Martins AM, Barreto SM, Pordeus IA. [Objective and subjective factors related to self-rated oral health among the elderly]. Cad Saude Publica. 2009 Feb;25(2):421-35

26. Steele JG, Sanders AE, Slade GD, Allen PF, Lahti S, Nuttall N, etal. How do age and tooth loss affect oral health impacts and quality of life? A study comparing two national samples. Community Dent Oral Epidemiol. 2004 Apr;32(2):107-14.

27. Gerritsen AE, Allen PF, Witter DJ, BronkhorstEM, Creugers NH. Tooth loss and oral health-related quality of life: a systematic review and meta-analysis. Health Qual Life Outcomes. 2010 Nov 5;8:126. doi:10.1186/1477-7525-8-126.

28. Esmeriz CE, Meneghim MC,Ambrosano GM. Self-perception of oral health in non-institutionalised elderly of Piracicaba city, Brazil. Gerodontology. 2012 Jun,29(2):e281-9. doi: 10.1111/j.1741-2358.2011.00464.x.

29. Hassel AJ, Steuker B, Rolko C, Keller L, Rammelsberg P,Nitschke I. Oral healthrelated quality of life of elderly Germans--comparison of GOHAl and OHIP-14. Community Dent Health. 2010 Dec;27(4):242-7.

30. El Osta N, Tubert-Jeannin S, Hennequin M, Bou Abboud Naaman N, El Osta L, Geahchan N. Comparison of the OHIP-14 and GOHAl as measures of oral health among elderly in Lebanon. Health Qual Life Outcomes. 2012 Oct 30:10:131. doi: 10.1186/1477-7525-10-131.

\section{Lígia Moura Burci}

(Corresponding address)

Postgraduate Program in Pharmaceutical Sciences (PPGCF)

Universidade Federal do Paraná (UFPR)

Av. Lothario Meissner, s/n, Jardim Botânico

Date submitted: 2019 0ct 01

81530-900 Curitiba, PR, Brazil. 